\title{
A NOTE ON MERTON'S PORTFOLIO SELECTION PROBLEM FOR THE SCHWARTZ MEAN-REVERSION MODEL
}

\author{
FRED ESPEN BENTH AND KENNETH HVISTENDAHL KARLSEN
}

\begin{abstract}
We analyze the classical Merton's portfolio optimization problem when the risky asset follows an exponential Ornstein-Uhlenbeck process, also known as the Schwartz mean-reversion dynamics. The corresponding Hamilton-Jacobi-Bellman equation is a two-dimensional nonlinear parabolic partial differential equation. We produce an explicit solution to this equation by reducing it to a simpler one-dimensional linear parabolic equation. This reduction is achieved through a Cole-Hopf type transformation, recently introduced in portfolio optimization theory by Zariphopoulou [9]. A verification argument is then used to prove that this solution coincides with the value function of the control problem. The optimal investment strategy is also given explicitly. On the technical side, the main problem we are facing here is the necessity to identify conditions on the parameters of the control problem ensuring uniform integrability of a family of random variables that roughly speaking are the exponentials of squared Wiener integrals.
\end{abstract}

\section{IntRodUCtION}

We consider the classical Merton's portfolio optimization problem when the risky asset follows an exponential Ornstein-Uhlenbeck process. This process was suggested by Schwartz [8] as a reasonable model for the spot price evolution of commodities like gold or coffee, and energies like oil, gas, and electricity (see also Clewlow and Strickland [2] or Pilipovic [6]), but could also be a model for the price of a bond or the interest-rate dynamics. In this paper we analyze the optimal investment problem for an agent in a financial market where the risky asset follows the price dynamics of Schwartz, and the risk preferences is described by a power utility (also known as HARA utility). This portfolio optimization problem is a variant of the classical Merton problem [3]. However, using the Schwartz dynamics rather than the classical geometric Brownian motion to model the risky asset price leads to some interesting problems that needs to be overcome in order to rigorously derive a solution.

We shall use the dynamic programming approach to derive an optimal investment strategy and the indirect utility. The main ingredient in the solution approach will be a transformation of Cole-Hopf type which reduces the dimension and removes the nonlinearity of the related Hamilton-Jacobi-Bellman (HJB henceforth) equation, enabling us to find an explicit solution. This transformation was introduced by Zariphpoulou [9], who applied it to a class of investment problems. Later the

Date: April 14, 2004.

Key words and phrases. Portfolio optimization, mean-reversion, dynamic programming, Hamilton-Jacobi-Bellman equation, Cole-Hopf transform, verification theorem.

This work was supported by the BeMatA program of the Research Council of Norway and the European network HYKE, funded by the EC as contract HPRN-CT-2002-00282. 
transform has proven useful in analyzing portfolio optimization and utility indifference pricing for stochastic volatility models, see Musiela and Zariphopoulou [4]. Pham [5] used an exponential transformation for the same type of control problems which works also in a multi-dimensional market. However, in the case of only one risky asset the transformation of Zariphpopoulou seems to be more efficient as it fully removes the nonlinearity, whereas the one employed in [5] does not.

The solution of the HJB equation is associated with the value function of the control problem using a verification theorem. The verification theorem also provides us with an explicit form of the optimal investment strategy. The main ingredient in the verification is to prove uniform integrability of the family of random variables generated by the value function evaluated at the optimal wealth process and the Schwartz dynamics stopped at exit times. By the nature of the candidate value function, this problem is essentially equivalent to the question of uniform integrability of a family of exponentials of squared Wiener integrals. Under certain conditions on the parameters of the control problem, we show that the family indeed is uniform integrable, thereby providing sufficient conditions for existence and uniqueness of a classical solution of the stochastic control problem.

It is worth while emphasizing that our candidate solution exists as a classical solution for general choices of parameters. This provides us with an upper bound for the value function, since the verification theorem tells us that any classical solution dominates the value function. To uniquely identify the candidate solution as the value function of the control problem, we need to impose additional conditions on the parameters, where one interpretation is the existence of a maximal investment horizon where this identification holds. The uniqueness is implied by the above mentioned uniform integrability, but can also be viewed in light of the well-known explosion/uniqueness condition for the initial value problem for the heat equation. Under explicit conditions on the growth rate of the initial condition, there exists a unique solution up to a certain time, at which the classical Green's function representation explodes. After the explosion time, infinitely many solutions exist. Our control problem possesses similar features.

Zariphopoulou [9] solved a class of portfolio optimization problems similar to ours using the transformation technique and a Feynman-Kac representation of the reduced HJB-equation. Using a verification theorem, the solution was associated to the value function of the control problem. However, the conditions on the drift of the price dynamics for the risky asset are too strong to be satisfied by the Schwartz dynamics. We can therefore not rely on her results when solving the stochastic control problem treated in this paper. Furthermore, in our specific case we are able to find an explicit solution to the reduced HJB-equation.

The explicit portfolio allocation rule depends on current time and price of the spot. However, as for Merton's classical problem [3], the current level of wealth does not affect the (proportional) investment decisions. The allocation can go negative for sufficiently small spot price levels. It is distributed according to an affine transformation of $\ln S_{t}$, meaning that the optimal investment process is Gaussian.

The paper is organized as follows: in Section 2 we state the portfolio optimization problem and the associated HJB-equation using the dynamic programming method. Furthermore, we reduce the dimension and remove the nonlinearity of the HJB equation by employing a Cole-Hopf type transformation. In Section 3 we derive an explicit solution to the reduced HJB-equation. A verification theorem for our 
control problem is proved in Section 4, along with a proof that the solution of the reduced HJB-equation is indeed the value function of the control problem. We end this section with some remarks on the nature of the solution and a discussion of the conditions on the parameters.

\section{The PORTFOLIO OPTIMIZATION PROBLEM}

We are given a complete probability space $\left(\Omega, \mathcal{F},\left\{\mathcal{F}_{t}\right\}_{t \in[0, T]}, P\right)$ satisfying the usual hypotheses, and let $B_{t}$ be a Brownian motion defined on it. The time horizon is assumed to be finite, $T<\infty$.

Let the price dynamics of the risky asset follow the Schwartz model

$$
d S_{t}=\alpha\left(\mu-\ln S_{t}\right) S_{t} d t+\sigma S_{t} d B_{t},
$$

where $S_{0}$ is the price observed in the market today and $\alpha, \mu, \sigma$ are constants. The speed of mean-reversion is modelled by $\alpha>0$, while the level is $\mu$. The volatility $\sigma$ is naturally assumed to be a positive constant. Note that $X_{t}=\ln S_{t}$ is an Ornstein-Uhlenbeck process

$$
d X_{t}=\alpha\left(\mu+\frac{\sigma^{2}}{2 \alpha}-X_{t}\right) d t+\sigma d B_{t}
$$

which has the explicit solution

$$
X_{t}=\mathrm{e}^{-\alpha t} \ln S_{0}+\left(\mu+\frac{\sigma^{2}}{2 \alpha}\right)\left(1-\mathrm{e}^{-\alpha t}\right)+\int_{0}^{t} \mathrm{e}^{\alpha(u-t)} d B_{u} .
$$

The risk-free investment (e.g., a bond) has the usual dynamics

$$
d R_{t}=r R_{t} d t, \quad R_{0}=1,
$$

with $r>0$ the (constant) continuously compounded risk-free interest rate. Now assume that the investor starts at time $t \leq T$ with initial wealth $W_{t}=w$. Given the spot price level $S_{t}=s$, the investor allocates a fraction $\pi_{u}, u \in[t, T]$, of the total wealth $W_{u}$ in the risky asset, where $\pi_{u}$ is a progressively measurable process. We shall consider Markov controls $\pi \equiv \pi(t, w, s)$, that is, controls on feedback form. After applying the self-financing hypothesis, the wealth dynamics reads

$$
d W_{u}^{\pi}=\left(\alpha \pi_{u}\left(\mu-\ln S_{u}\right)+\left(1-\pi_{u}\right) r\right) W_{u}^{\pi} d u+\sigma \pi_{u} W_{u}^{\pi} d B_{u}, \quad t<u \leq T .
$$

We call $\pi_{u}$ admissible if there exists a unique positive wealth process solving (2.2) for which

$$
\int_{t}^{T} \pi_{u}^{2}\left(W_{u}^{\pi}\right)^{2} d u<\infty .
$$

The set of admissible controls is denoted $\mathcal{A}_{t}$, where the subscript $t$ indicates the initial time for the investment, given that $W_{t}^{\pi}=w$ and $S_{t}=s$. When it is clear from the context we shall frequently omit the superscript $\pi$ and simply write $W_{u}$ for the wealth process.

Remark. Zariphopoulou [9] assumes Lipschitz continuous drift and diffusion functions. In our case we have a drift being the function $\alpha(\mu-\ln s) s$, which is not Lipschitz continuous. Moreover, in [9] it is supposed that the square of the drift (less the risk-free interest rate) divided by the volatility should be bounded. In the Schwartz model this entails a boundedness restriction on $(\mu-r-\ln s)^{2} / \sigma^{2}$, which obviously does not hold. Finally, it is assumed that the drift divided by $s$ should 
be greater than the risk free interest rate $r$, which for the Schwartz model means that $(\mu-\ln s)>r$. This can not be true either. In conclusion, the drift structure of the Schwartz model clearly violates the assumptions made in [9].

The investor is assumed to have risk preferences given by a power utility $U(w)=$ $\gamma^{-1} w^{\gamma}$ where $\gamma \in(0,1)$. The optimization problem faced by the investor is: find an admissible trading strategy $\pi_{t}$ such that utility derived from wealth is maximized at the final time $T$. The value function is defined as

$$
V(t, w, s)=\sup _{\pi \in \mathcal{A}_{t}} \mathbb{E}^{t, w, s}\left[U\left(W_{T}^{\pi}\right)\right],
$$

where the supremum is taken over all admissible strategies $\mathcal{A}_{t}$. The operator $\mathbb{E}^{t, w, s}$ means the expectation conditioned on $W_{t}^{\pi}=w$ and $S_{t}=s$.

Using the classical dynamic programming principle we derive the HJB equation for the value function:

$$
\begin{aligned}
\partial_{t} v+\max _{\pi}\left\{((\alpha(\mu-\ln s)-r) \pi+r) w \partial_{w} v+\frac{1}{2} \sigma^{2} \pi^{2} w^{2} \partial_{w w} v+\sigma^{2} \pi w s \partial_{w s} v\right\} \\
\quad+\alpha(\mu-\ln s) s \partial_{s} v+\frac{1}{2} \sigma^{2} s^{2} \partial_{s s} v=0 .
\end{aligned}
$$

Here (and elsewhere) $\pi$ runs over all real numbers. The natural terminal and boundary conditions are

$$
v(T, w, s)=U(w), \quad v(t, 0, s)=0 .
$$

Introduce from now on the notation

$$
\begin{aligned}
\mathcal{L}^{\pi} v=\partial_{t} v+ & ((\alpha(\mu-\ln s)-r) \pi+r) w \partial_{w} v+\frac{1}{2} \sigma^{2} \pi^{2} w^{2} \partial_{w w} v+\sigma^{2} \pi w s \partial_{w s} v \\
& +\alpha(\mu-\ln s) s \partial_{s} v+\frac{1}{2} \sigma^{2} s^{2} \partial_{s s} v
\end{aligned}
$$

Then the HJB equation can be compactly written as

$$
\max _{\pi} \mathcal{L}^{\pi} v=0 \text {. }
$$

Assuming there exists a sufficiently smooth solution $v$ to the HJB equation, the first order condition for an optimal $\pi$ gives

$$
\pi^{\star}=-\frac{(\alpha(\mu-\ln s)-r) \partial_{w} v+\sigma^{2} s \partial_{w s} v}{\sigma^{2} w \partial_{w w} v}
$$

while the second order condition is $\sigma^{2} w^{2} \partial_{w w} v \leq 0$, which in other words says that $v$ must be concave as a function of $w$.

Inserting the expression for an optimal $\pi^{\star}$ yields after some routine manipulations the following two-dimensional nonlinear parabolic equation

$$
\begin{aligned}
\partial_{t} v+r w \partial_{w} V & +\alpha(\mu-\ln s) s \partial_{s} v+\frac{1}{2} \sigma^{2} s^{2} \partial_{s s} v-\frac{1}{2} \sigma^{2} s^{2} \frac{\left(\partial_{w s} v\right)^{2}}{\partial_{w w} v} \\
& -\frac{(\alpha(\mu-\ln s)-r)^{2}}{2 \sigma^{2}} \frac{\left(\partial_{w} v\right)^{2}}{\partial_{w w} v}-(\alpha(\mu-\ln s)-r) s \frac{\partial_{w} v \partial_{w s} v}{\partial_{w w} v}=0 .
\end{aligned}
$$

The goal is to construct an explicit solution to (2.6) with terminal and boundary conditions $v(T, w, s)=\gamma^{-1} w^{\gamma}$ and $v(t, 0, s)=0$. However, as it stands, it is not easy to solve (2.6). Therefore we shall first reduce the dimension and remove the nonlinearities in the HJB equation (2.6). Motivated from Merton's solution [3] and 
the nonlinear transformation introduced by Zariphopoulou [9], we make the ansatz that the solution of (2.6) takes the form

$$
v(t, w, s)=\gamma^{-1} w^{\gamma} g(t, s)^{1-\gamma}
$$

for some function $g(t, s)$ to be determined. Observe first that $g(T, s)=1$.

Inserting $v(t, w, s)$ into (2.6) produces the following equation for $g$ :

$$
\begin{aligned}
& \partial_{t} g+\frac{\alpha}{1-\gamma}\left(\mu-\frac{\gamma r}{\alpha}-\ln s\right) s \partial_{s} g+\frac{1}{2} \sigma^{2} s^{2} \partial_{s s} g \\
& \quad+\left(\frac{r \gamma}{1-\gamma}+\frac{\gamma(\alpha(\mu-\ln s)-r)^{2}}{2 \sigma^{2}(1-\gamma)^{2}}\right) g=0 \\
& g(T, s)=1,
\end{aligned}
$$

which indeed is a one-dimensional linear parabolic equation with a particular source term. We shall construct an explicit solution to (2.7) in the next section.

Remark. Pham [5] proposes a transform which in our context reads $v(t, w, s)=$ $\gamma^{-1} w^{\gamma} \exp (-\phi(t, s))$. Direct differentiation leads to a semilinear parabolic partial differential equation for $\phi$ :

$$
\begin{aligned}
-\partial_{t} \phi & +\left(r \gamma+\frac{\gamma}{2 \sigma^{2}(1-\gamma)}(r-\alpha(\mu-\ln s))^{2}\right) \\
& -\left(\frac{r \gamma}{1-\gamma}+\frac{1-2 \gamma}{1-\gamma} \alpha(\mu-\ln s)\right) s \partial_{s} \phi-\frac{1}{2} \sigma^{2} s^{2} \partial_{s s} \phi+\frac{1}{2} \sigma^{2} s^{2}\left(\partial_{s} \phi\right)^{2}=0
\end{aligned}
$$

with terminal condition $\phi(T, s)=0$. In Pham [5] the function $\phi(t, s)$ is represented as the solution of a control problem.

\section{Construction of a solution to the reduced HJB equation}

In this section we construct an explicit solution to the linear equation $(2.7)$. Following the idea of Zariphopoulou [9] we could use a Feynman-Kac approach, that is, represent the solution $g(t, s)$ of $(2.7)$ as an expectation operator of a stochastic process $\widetilde{S}_{t}$, where

$$
\left.d \widetilde{S}_{t}=\frac{\alpha}{1-\gamma}\left(\mu-\frac{\gamma r}{\alpha}-\ln \widetilde{S}_{t}\right)\right) \widetilde{S}_{t} d t+\sigma \widetilde{S}_{t} d B_{t}
$$

Note that $\widetilde{S}_{t}$ is a mean-reversion model with speed of mean-reversion given by $\alpha /(1-\gamma)$, while the level is $\mu-\gamma r / \alpha$. The volatility is the same as for $S_{t}$, namely $\sigma$.

We can easily derive an explicit solution of $\widetilde{S}_{t}$ via its $\log$ arithm $\widetilde{X}_{t}=\ln \widetilde{S}_{t}$, using that this is an Ornstein-Uhlenbeck process. Moreover, the Feynman-Kac formula yields the following representation

$$
g(t, s)=\mathbb{E}\left[\exp \left(\frac{\gamma}{1-\gamma} \int_{t}^{T}\left[r+\frac{\left(\alpha\left(\mu-\ln \widetilde{S}_{u}\right)-r\right)^{2}}{2 \sigma^{2}(1-\gamma)}\right] d u\right) \mid \widetilde{S}_{t}=s\right] .
$$

It turns out that this expectation admits an explicit representation.

Indeed, it is reasonable to guess that the solution $g(t, s)$ of $(2.7)$ takes the form

$$
g(t, s)=\exp \left(f_{0}(t)+f_{1}(t) \ln s+f_{2}(t)(\ln s)^{2}\right)
$$

for some functions $f_{i}(t), i=0,1,2$, that have to be determined. In the rest of this section we will derive differential equations satisfied by $f_{i}(t), i=0,1,2$, and find the corresponding solutions. 
Lemma 3.1. The functions $f_{i}:[0, T] \rightarrow \mathbb{R}, i=0,1,2$, in (3.2) satisfy the following system of ordinary differential equations:

$$
f_{0}^{\prime}(t)=-\left(r+\frac{\alpha \mu-r}{1-\gamma}\right) f_{1}(t)-\sigma^{2} f_{2}(t)+\frac{2 \alpha r \mu-r^{2}-\alpha^{2} \mu^{2} \gamma}{2 \sigma^{2}(1-\gamma)^{2}}-\frac{r \gamma}{1-\gamma},
$$

$$
f_{1}^{\prime}(t)=\left(\frac{\alpha}{1-\gamma}-2 \sigma^{2} f_{2}(t)\right) f_{1}(t)+\frac{\alpha(\alpha \mu \gamma-r)}{\sigma^{2}(1-\gamma)^{2}}+\left(\sigma^{2}-2 r-\frac{2(\alpha \mu-r)}{1-\gamma}\right) f_{2}(t),
$$

$$
f_{2}^{\prime}(t)=-2 \sigma^{2}\left(f_{2}(t)-\frac{\alpha}{2 \sigma^{2}(1-\gamma)}\right)^{2}+\frac{\alpha^{2}}{2 \sigma^{2}(1-\gamma)}
$$

where $f_{i}(T)=0$ for $i=0,1,2$.

Proof. First observe that since $g(T, s)=1$, we have

$$
f_{0}(T)+f_{1}(T) \ln s+f_{2}(T)(\ln s)^{2}=0 .
$$

Necessarily, by varying $s$, we obtain $f_{i}(T)=0, i=0,1,2$.

Inserting $g(t, s)$ into $(2.7)$ we find

$$
\begin{aligned}
f_{0}^{\prime}(t) & +f_{1}^{\prime}(t) \ln s+f_{2}^{\prime}(t)(\ln s)^{2} \\
& +\left(\frac{r \gamma}{1-\gamma}+\frac{\gamma(\alpha(\mu-\ln s)-r)^{2}}{2 \sigma^{2}(1-\gamma)^{2}}\right) \\
& +\frac{1}{2} \sigma^{2}\left(\left(f_{1}(t)+2 f_{2}(t) \ln s\right)^{2}-f_{1}(t)+2 f_{2}(t)(1-\ln s)\right) \\
& +\left(r+\frac{\alpha(\mu-\ln s)-r}{1-\gamma}\right)\left(f_{1}(t)+2 f_{2}(t) \ln s\right)=0 .
\end{aligned}
$$

Collecting all terms with $\ln s$ and $(\ln s)^{2}$, we observe that these must be equal to zero since $s$ varies. Similarly, the collection of factors independent of $s$ must be zero. Hence, after some reorganization, we end up with the desired system of differential equations.

Observe that the three differential equations for $f_{0}, f_{1}$, and $f_{2}$ are coupled. The coupling are recursive, in the sense that the equation for $f_{2}$ is autonomous, while we can go recursively backwards in order to find $f_{1}$ and $f_{0}$, given that we know $f_{2}$.

Lemma 3.2. The solution $f_{2}:[0, T] \rightarrow \mathbb{R}$ to the nonlinear $O D E$ (3.5) is given by

$$
f_{2}(t)=\frac{\alpha \gamma}{2 \sigma^{2}(1-\gamma)} \frac{\sinh \left(\frac{\alpha}{\sqrt{1-\gamma}}(T-t)\right)}{\sinh \left(\frac{\alpha}{\sqrt{1-\gamma}}(T-t)\right)+\sqrt{1-\gamma} \cosh \left(\frac{\alpha}{\sqrt{1-\gamma}}(T-t)\right)} .
$$

Moreover, $f_{2}(t)$ is a nonincreasing function on $[0, T]$.

Proof. That $f_{2}$ solves (3.5) goes by direct verification. Differentiating the expression of $f_{2}$, or inserting the explicit representation of $f_{2}$ into the right-hand side of (3.5), shows that $f_{2}^{\prime}(t)<0$ for $t \in(0, T)$. 
Obviously $f_{2}$ is strictly positive on $[0, T)$ since it is nonincreasing and $f_{2}(T)=0$. Also, the denominator of $f_{2}(t)$ is strictly positive when $t \in[0, T]$ for all $T>0$, and thus $f_{2}(t)$ does not explode. The positivity of $f_{2}(t)$ creates some delicate problems when we want to use a verification argument to identify our solution candidate with the value function $V$. We need to handle exponential moments of the squares of an Ornstein-Uhlenbeck process. In the next section these aspects are considered.

Lemma 3.3. The solutions $f_{0}, f_{1}:[0, T] \rightarrow \mathbb{R}$ to the ordinary differential equations (3.3), (3.4) are given by

$$
\begin{aligned}
& f_{0}(t)=\int_{t}^{T}\{(r+\left.\left.\frac{\alpha \mu-r}{1-\gamma}\right) f_{1}(s)+\sigma^{2} f_{2}(s)\right\} d s \\
&+\left(\frac{r \gamma}{1-\gamma}-\frac{2 \alpha \mu r-r^{2}-\alpha^{2} \mu^{2} \gamma}{2 \sigma^{2}(1-\gamma)^{2}}\right)(T-t), \\
& f_{1}(t)=-\int_{t}^{T} b(s) \exp \left(-\int_{t}^{s} a(u) d u\right) d s
\end{aligned}
$$

where

$$
a(t)=\frac{\alpha}{1-\gamma}-2 \sigma^{2} f_{2}(t), \quad b(t)=\frac{\alpha(\alpha \mu \gamma-r)}{\sigma^{2}(1-\gamma)^{2}}+\left(\sigma^{2}-2 r-\frac{2(\alpha \mu-r)}{1-\gamma}\right) .
$$

Proof. Follows by direct differentiation.

The following theorem summarizes our findings.

Theorem 3.4. A solution to the HJB equation (2.6) is

$$
v(t, w, s)=\gamma^{-1} w^{\gamma} \exp \left(h_{0}(t)+h_{1}(t) \ln s+h_{2}(t)(\ln s)^{2}\right),
$$

where $h_{i}(t)=(1-\gamma) f_{i}(t)$ and $f_{i}, i=1,2,3$, are defined in Lemmas 3.2 and 3.3.

Note that $h_{2}$ is a positive function on $[0, T)$.

\section{Solution of the portfolio optimization problem}

We present two propositions which together serve as a verification theorem for candidate solutions of the HJB equation. The results and proofs are classical.

Proposition 4.1. Assume $\phi \in C^{2}\left([0, T) \times \mathbb{R}_{+}^{2}\right)$ is a non-negative function such that $\mathcal{L}^{\pi} \phi \leq 0, \phi(T, w, s)=U(w)$ and $\phi(t, 0, s)=0$ for every admissible control $\pi$. Then

$$
\phi(t, w, s) \geq \mathbb{E}^{t, w, s}\left[U\left(W_{T}^{\pi}\right)\right]
$$

Proof. Assume $\pi$ is an admissible control, and let $\left\{\tau_{n}\right\}_{n}$ be a localizing sequence of stopping times for the local semimartingale $\left(W_{u}^{\pi}, S_{u}\right)$ starting in $(w, s)$ at time $t$. Introducing the stopping times $T_{n}=\min \left(T, \tau_{n}\right)$, we get from Itô's Formula

$$
\begin{aligned}
& \phi\left(T_{n}, W_{T_{n}}^{\pi}, S_{T_{n}}\right)= \phi(t, w, s)+\int_{t}^{T_{n}} \mathcal{L}^{\pi} \phi\left(u, W_{u}^{\pi}, S_{u}\right) d u \\
& \quad+\sigma \int_{t}^{T_{n}}\left(\pi_{u} W_{u}^{\pi} \partial_{w}+S_{u} \partial_{s}\right) \phi\left(u, W_{u}^{\pi}, S_{u}\right) d B_{u} \\
& \leq \phi(t, w, s)+\sigma \int_{t}^{T_{n}}\left(\pi_{u} W_{u}^{\pi} \partial_{w}+S_{u} \partial_{s}\right) \phi\left(u, W_{u}^{\pi}, S_{u}\right) d B_{u}
\end{aligned}
$$


The Itô integral is a martingale, hence, after taking expectations, we find

$$
\phi(t, w, s) \geq \mathbb{E}^{t, w, s}\left[\phi\left(T_{n}, W_{T_{n}}^{\pi}, S_{T_{n}}\right)\right] .
$$

Since $\phi$ is non-negative, $\left\{\phi\left(T_{n}, W_{T_{n}}^{\pi}, S_{T_{n}}\right)\right\}_{n}$ is a sequence of non-negative measurable functions. Since

$$
\lim _{n \rightarrow \infty} \phi\left(T_{n}, W_{T_{n}}^{\pi}, S_{T_{n}}\right)=\phi\left(T, W_{T}^{\pi}, S_{T}\right)=U\left(W_{T}^{\pi}\right), \quad \text { a.s. },
$$

Fatou's lemma yields

$$
\begin{aligned}
\mathbb{E}^{t, w, s}\left[U\left(W_{T}^{\pi}\right)\right] & =\mathbb{E}^{t, w, s}\left[\liminf _{n \rightarrow \infty} \phi\left(T_{n}, W_{T_{n}}^{\pi}, S_{T_{n}}\right)\right] \\
& \leq \liminf _{n \rightarrow \infty} \mathbb{E}^{t, w, s}\left[\phi\left(T_{n}, W_{T_{n}}^{\pi}, S_{T_{n}}\right)\right] \\
& \leq \phi(t, w, s) .
\end{aligned}
$$

This proves the proposition.

Proposition 4.2. Let $\phi \in C^{2}\left([0, T) \times \mathbb{R}_{+}^{2}\right)$ be a non-negative function such that the family of random variables $\left\{\phi\left(\tau, W_{\tau}^{\pi^{\star}}, S_{\tau}\right)\right\}_{\tau}$ is uniformly integrable, where $\pi^{\star}$ is an admissible control with the property $\mathcal{L}^{\pi^{\star}} \phi=0$ and $t \leq \tau \leq T$ is a stopping time for the process $\left(W_{u}^{\pi^{\star}}, S_{u}\right)$ starting in $(w, s)$ at time $t$. If furthermore $\phi(T, w, s)=U(w)$, $\phi(t, 0, s)=0$ and $\mathcal{L}^{\pi} \phi \leq 0$ for all admissible controls $\pi$, then

$$
\phi(t, w, s)=V(t, w, s) \quad \forall(t, w, s) \in[0, T] \times \mathbb{R}_{+}^{2} .
$$

Proof. Inspecting the proof of Proposition 4.1, we see that the uniform integrability of the family $\left\{\phi\left(\tau, W_{\tau}^{\pi^{\star}}, S_{\tau}\right)\right\}$, $\tau$ stopping times, yields that

$$
\begin{aligned}
\mathbb{E}^{t, w, s}\left[U\left(W_{T}^{\pi^{\star}}\right)\right] & =\mathbb{E}\left[\lim _{n \rightarrow \infty} \phi\left(T_{n}, W_{T_{n}}^{\pi^{\star}}, S_{T_{n}}\right)\right] \\
& =\lim _{n \rightarrow \infty} \mathbb{E}^{t, w, s}\left[\phi\left(T_{n}, W_{T_{n}}^{\pi^{\star}}, S_{T_{n}}\right)\right],
\end{aligned}
$$

where $T_{n}$ is the minimum of $T$ and the localizing sequence $\left\{\tau_{n}\right\}_{n}$ for $\left(W_{u}^{\pi^{\star}}, S_{u}\right)$ starting in $(w, s)$ at time $t$. Furthermore, since $\mathcal{L}^{\pi^{*}} \phi=0$, we get

$$
\mathbb{E}^{t, w, s}\left[U\left(W_{T}^{\pi^{\star}}\right)\right]=\phi(t, w, s),
$$

and hence, by Proposition 4.1, $\phi(t, w, s)=V(t, w, s)$ for all $(t, w, s) \in[0, T] \times$ $\mathbb{R}_{+}^{2}$.

To verify that the candidate solution $v$ in Theorem 3.4 is the desired value function (2.4), we need to show that $v$ satisfies the uniform integrability condition in Proposition 4.2. To this end, we must make sure that terms involving $\left(\ln S_{u}\right)^{2}$ and their time integrals are exponentially integrable, which is equivalent of asking for exponential integrability of the square of an Ornstein-Uhlenbeck process and its time integral. This is true only under certain restrictions on the coefficients, since basically we are calculating the expectation of the exponential of the square of a normal random variable which may become infinite if parameters are not properly scaled. In the following lemma we state sufficient conditions to ensure such exponential integrability. 
Lemma 4.3. If $\lambda$ is a constant such that

$$
\lambda<\frac{\alpha}{2 \sigma^{2}(T-t)}
$$

then

$$
\mathbb{E}^{t, s}\left[\exp \left\{\lambda \int_{t}^{T}\left(\ln S_{u}\right)^{2} d u\right\}\right]<\infty .
$$

Proof. First recall that $X_{u}:=\ln S_{u}$ is an Ornstein-Uhlenbeck process where

$$
X_{u}=\mathrm{e}^{-\alpha(u-t)} \ln S_{t}+\left(\mu+\sigma^{2} / 2 \alpha\right)\left(1-\mathrm{e}^{-\alpha(u-t)}\right)+\sigma \int_{t}^{u} \mathrm{e}^{-\alpha(u-v)} d B_{v} .
$$

Hence,

$$
\mathbb{E}^{t, s}\left[\exp \left\{\lambda \int_{t}^{T}\left(\ln S_{u}\right)^{2} d u\right\}\right] \leq c \mathbb{E}^{t, s}\left[\exp \left\{2 \sigma^{2} \lambda \int_{t}^{T} Z_{s}^{2} d s\right\}\right]
$$

where

$$
Z_{u} \sim \mathcal{N}\left(0, \frac{1}{2 \alpha}\left(1-\mathrm{e}^{-2 \alpha(u-t)}\right)\right)
$$

given that we start in zero at time $t$. A straightforward estimation using Hölder's inequality with $p=n$ and $q=n / n-1$, and the normality of $Z_{u}$, yields

$$
\begin{aligned}
\mathbb{E}\left[\exp \left\{2 \sigma^{2} \lambda \int_{t}^{T} Z_{s}^{2} d s\right\}\right] & =\sum_{n=0}^{\infty} \frac{\left(2 \sigma^{2} \lambda\right)^{n}}{n !} \mathbb{E}^{t, 0}\left[\left(\int_{t}^{T} Z_{u}^{2} d u\right)^{n}\right] \\
& \leq(T-t)^{-1} \sum_{n=0}^{\infty} \frac{\left(2 \sigma^{2} \lambda(T-t)\right)^{n}}{n !} \int_{t}^{T} \mathbb{E}^{t, 0}\left[Z_{u}^{2 n}\right] d u \\
& \leq \frac{1}{\sqrt{\pi}(T-t)} \sum_{n=0}^{\infty} \frac{\left(2 \sigma^{2} \lambda(T-t) \alpha^{-1}\right)^{n}}{n !} \Gamma\left(n+\frac{1}{2}\right) .
\end{aligned}
$$

The series is finite whenever $2 \sigma^{2} \lambda(T-t)<\alpha$, which holds thanks to (4.1).

The following theorem is the main result of this paper, linking the solution of the HJB equation to the value function of the control problem under certain integrability conditions. It is the basic content of the proof to derive sufficient conditions on the parameters of the control problem ensuring uniform integrability, as required by Proposition 4.2.

Theorem 4.4. Assume that there exist two positive constants $\epsilon$ and $\delta$ such that

$$
\begin{gathered}
\left(2(1+\epsilon) h_{2}(t)+\delta\right)\left(\delta+\sigma^{2} \exp (-2 \alpha t)\right)<\alpha /(\exp (2 \alpha T)-\exp (2 \alpha t)), \\
8(1+\epsilon) \gamma\left(\delta \sigma^{2}(1-\gamma)+\alpha^{2}\right)<\alpha(1-\gamma) /(T-t),
\end{gathered}
$$

and

$$
64(1+\epsilon)^{2} \gamma^{2}\left(\delta \sigma^{2}(1-\gamma)^{2}+\alpha^{2}+4 \sigma^{4} h_{2}(t)\right)<\alpha(1-\gamma)^{2} /(T-t) .
$$

Then the value function of the control problem is

$$
V(t, w, s)=\gamma^{-1} w^{\gamma} \exp \left(h_{0}(t)+h_{1}(t) \ln s+h_{2}(t)(\ln s)^{2}\right),
$$


where $h_{i}(t), i=0,1,2$ are given in Theorem 3.4. Moreover, the optimal investment strategy is

$$
\pi^{\star}(t, s)=\frac{\alpha \mu-r+\sigma^{2} h_{1}(t)}{\sigma^{2}(1-\gamma)}+\frac{2 \sigma^{2} h_{2}(t)-\alpha}{\sigma^{2}(1-\gamma)} \ln s
$$

Proof. Denote by $v$ the candidate solution from Theorem 3.4. We must show that $V(t, w, s)=v(t, w, s)$. From the first order condition we easily see that $\pi^{\star}$ has the property $\mathcal{L}^{\pi^{\star}} v=0$. To simplify the estimations, introduce the following notations:

$$
\begin{aligned}
& \alpha_{0}(t)=r+(\alpha \mu-r) \frac{\alpha \mu-r+\sigma^{2} h_{1}(t)}{\sigma^{2}(1-\gamma)}, \\
& \alpha_{1}(t)=\frac{2(\alpha \mu-r) \sigma^{2} h_{2}(t)-\alpha \sigma^{2} h_{1}(t)-2 \alpha(\alpha \mu-r)}{\sigma^{2}(1-\gamma)}, \\
& \alpha_{2}(t)=\frac{\alpha^{2}-2 \sigma^{2} \alpha h_{2}(t)}{\sigma^{2}(1-\gamma)} \\
& \sigma_{0}(t)=\frac{\alpha \mu-r+\sigma^{2} h_{1}(t)}{\sigma(1-\gamma)} \\
& \sigma_{1}(t)=\frac{2 \sigma^{2} h_{2}(t)-\alpha}{\sigma(1-\gamma)} .
\end{aligned}
$$

It is straightforward to see that the wealth dynamics for the control $\pi^{\star}$ becomes

$$
\begin{gathered}
d W_{t}^{\star}=\left(\alpha_{0}(t)+\alpha_{1}(t) \ln S_{t}+\alpha_{2}(t)\left(\ln S_{t}\right)^{2}\right) W_{t}^{\star} d t \\
+\left(\sigma_{0}(t)+\sigma_{1}(t) \ln S_{t}\right) W_{t}^{\star} d B_{t},
\end{gathered}
$$

which we easily see has the following unique positive solution:

$$
\begin{gathered}
W_{s}^{\star}=w \exp \left\{\int_{t}^{s} \alpha_{0}(u)-\frac{1}{2} \sigma_{0}^{2}(u)+\left(\alpha_{1}(u)-\sigma_{0}(u) \sigma_{1}(u)\right) \ln S_{u}\right. \\
+\left(\alpha_{2}(u)-\frac{1}{2} \sigma_{1}^{2}(u)\right)\left(\ln S_{u}\right)^{2} d u \\
\left.+\int_{t}^{s}\left(\sigma_{0}(u)+\sigma_{1}(u) \ln S_{u}\right) d B_{u}\right\}
\end{gathered}
$$

for $t \leq s \leq T$ and $W_{t}^{\star}=w$. Note in particular that this implies that $\pi^{\star}$ is an admissible control.

To apply Proposition 4.2 we must prove uniformly integrability of the family $\left\{v\left(\tau, W_{\tau}^{\star}, S_{\tau}\right)\right\}_{\tau}$. Recall that $v(t, w, s)=\gamma^{-1} w^{\gamma} g(t, s)^{1-\gamma}$, where

$$
g(t, s)^{1-\gamma}=\exp \left(h_{0}(t)+h_{1}(t) \ln s+h_{2}(t)(\ln s)^{2}\right) .
$$

Let $\epsilon>0$ and $t \leq \tau \leq T$ be a stopping time. Applying the Cauchy-Schwartz inequality we find that

$$
\begin{aligned}
& \mathbb{E}^{t, w, s} {\left[v^{1+\epsilon}\left(\tau, W_{\tau}^{\star}, S_{\tau}\right)\right] } \\
& \leq \gamma^{-(1+\epsilon)} \mathbb{E}^{t, w, s}\left[\left(W_{\tau}^{\star}\right)^{2(1+\epsilon) \gamma}\right]^{1 / 2} \mathbb{E}^{t, s}\left[g^{2(1+\epsilon)(1-\gamma)}\left(\tau, S_{\tau}\right)\right]^{1 / 2} .
\end{aligned}
$$

We shall estimate separately the two expectations on the right-hand side. The latter becomes

$$
\mathbb{E}^{t, s}\left[g^{2(1+\epsilon)(1-\gamma)}\left(\tau, S_{\tau}\right)\right]^{1 / 2}
$$




$$
\leq c \mathbb{E}^{t, s}\left[\exp \left\{2(1+\epsilon) h_{1}(\tau) \ln S_{\tau}+2(1+\epsilon) h_{2}(\tau)\left(\ln S_{\tau}\right)^{2}\right\}\right]^{1 / 2},
$$

where $c$ is some positive constant (which may change from line to line in what follows). Let $\delta>0$ be a constant. Then by Young's inequality it follows that

$$
2(1+\epsilon) h_{1}(\tau) \ln S_{\tau} \leq c+\delta\left(\ln S_{\tau}\right)^{2},
$$

where $c$ depends on the maximum of $h_{1}(u)$ for $t \leq u \leq T$ and $\delta$. Thus we have

$$
\mathbb{E}^{t, s}\left[g^{2(1+\epsilon)(1-\gamma)}\left(\tau, S_{\tau}\right)\right]^{1 / 2} \leq c \mathbb{E}^{t, s}\left[\exp \left\{\left(2(1+\epsilon) h_{2}(\tau)+\delta\right)\left(\ln S_{\tau}\right)^{2}\right\}\right]^{1 / 2} .
$$

From Lemma 3.2 we know that $f_{2}$, and thus $h_{2}$ is a nonincreasing function. Defining $\lambda:=2(1+\epsilon) h_{2}(t)+\delta$ therefore gives

$$
\mathbb{E}^{t, s}\left[g^{2(1+\epsilon)(1-\gamma)}\left(\tau, S_{\tau}\right)\right]^{1 / 2} \leq c \mathbb{E}^{t, s}\left[\exp \left\{\lambda\left(\ln S_{\tau}\right)^{2}\right\}\right]^{1 / 2} .
$$

Recall that

$$
\ln S_{\tau}=\mathrm{e}^{-\alpha(\tau-t)} \ln s+\left(\mu-\sigma^{2} / 2 \alpha\right)+\sigma \mathrm{e}^{-\alpha \tau} \int_{t}^{\tau} \mathrm{e}^{\alpha u} d B_{u} .
$$

Therefore, using the same argument as above, we find

$$
\mathbb{E}^{t, s}\left[g^{2(1+\epsilon)(1-\gamma)}\left(\tau, S_{\tau}\right)\right]^{1 / 2} \leq c \mathbb{E}^{t, s}\left[\exp \left\{\lambda\left(\delta+\sigma^{2} \mathrm{e}^{-2 \alpha t}\right)\left(\int_{t}^{\tau} \mathrm{e}^{\alpha u} d B_{u}\right)^{2}\right\}\right]^{1 / 2} .
$$

Now, since $\int_{t}^{s} \exp (\alpha u) d B_{u}$ is obviously a martingale, we have that for any positive constant $k$

$$
Z_{s}:=\exp \left\{k\left(\int_{t}^{s} \mathrm{e}^{\alpha u} d B_{u}\right)^{2}\right\}
$$

is a positive submartingale by Jensen's inequality since $x \mapsto \exp \left(k x^{2}\right)$ is a convex function, provided that $Z_{s} \in L^{1}(P)$ for all $s \in[t, T]$. However, since $\int_{t}^{s} \exp (\alpha u) d B_{u}$ is a centered normal random variable with variance $(\exp (2 \alpha s)-\exp (2 \alpha t)) / 2 \alpha$, $Z_{s} \in L^{1}(P)$ for all $t \leq s \leq T$ exactly when $k<\alpha /(\exp (2 \alpha T)-\exp (2 \alpha t))$. Letting

$$
k=\lambda\left(\delta+\sigma^{2} \exp (-2 \alpha t)\right),
$$

we see that this is satisfied by condition (4.2).

We estimate using Doob's martingale inequality (see e.g. Protter [7, Thm. 20])

$$
\begin{aligned}
& \mathbb{E}[\exp \left.\left\{k\left(\int_{t}^{\tau} \mathrm{e}^{\alpha u} d B_{u}\right)^{2}\right\}\right] \\
& \leq\left\|\exp \left\{\frac{k}{1+\delta}\left(\int_{t}^{\tau} \mathrm{e}^{\alpha u} d B_{u}\right)^{2}\right\}\right\|_{1+\delta}^{1+\delta} \\
&\left.\leq\left\|\sup _{t \leq s \leq T} \exp \left\{\frac{k}{1+\delta}\left(\int_{t}^{s} \mathrm{e}^{\alpha u} d B_{u}\right)^{2}\right\}\right\|_{1+\delta}^{1+\delta}\right\} \\
& \leq\left(1+\frac{1}{\delta}\right)^{1+\delta} \sup _{t \leq s \leq T} \mathbb{E}\left[\exp \left\{k\left(\int_{t}^{s} \mathrm{e}^{\alpha u} d B_{u}\right)^{2}\right\}\right]^{1+\delta} \\
& \leq c .
\end{aligned}
$$


Here, $\|\cdot\|_{p}, p>0$, denotes the norm in $L^{p}(P)$ and $c$ is a positive constant independent of the stopping time $\tau$. It follows that

$$
\mathbb{E}^{t, s}\left[g^{2(1+\epsilon)(1-\gamma)}\left(\tau, S_{\tau}\right)\right]^{1 / 2} \leq c,
$$

where $c$ is independent of $\tau$.

We continue with showing that the first expectation on the right-hand side in (4.7) is finite. By the Cauchy-Schwartz inequality

$$
\begin{aligned}
& \mathbb{E}^{t, w, s}\left[\left(W_{\tau}^{\star}\right)^{2(1+\epsilon) \gamma}\right] \\
& \leq c \mathbb{E}^{t, s} {\left[\operatorname { e x p } \left\{2(1+\epsilon) \gamma \int_{t}^{\tau}\left(\alpha_{1}(u)-\sigma_{0}(u) \sigma_{1}(u)\right) \ln S_{u}\right.\right.} \\
&+\left.\left.\left(\alpha_{2}(u)-\frac{1}{2} \sigma_{1}^{2}(u)\right)\left(\ln S_{u}\right)^{2} d u+2(1+\epsilon) \gamma \int_{t}^{\tau}\left(\sigma_{0}(u)+\sigma_{1}(u) \ln S_{u}\right) d B_{u}\right\}\right] \\
& \leq c \mathbb{E}^{t, s} {\left[\operatorname { e x p } \left\{4(1+\epsilon) \gamma \int_{t}^{\tau}\left(\alpha_{1}(u)-\sigma_{0}(u) \sigma_{1}(u)\right) \ln S_{u}\right.\right.} \\
&+\left.\left.\left(\alpha_{2}(u)-\frac{1}{2} \sigma_{1}^{2}(u)\right)\left(\ln S_{u}\right)^{2} d u\right\}\right]^{1 / 2} \\
& \times \mathbb{E}^{t, s}\left[\exp \left\{4(1+\epsilon) \gamma \int_{t}^{\tau}\left(\sigma_{0}(u)+\sigma_{1}(u) \ln S_{u}\right) d B_{u}\right\}\right]^{1 / 2} .
\end{aligned}
$$

Considering the first integral, we estimate by Young's inequality

$$
\left(\alpha_{1}(u)-\sigma_{0}(u) \sigma_{1}(u)\right) \ln S_{u} \leq c+\delta\left(\ln S_{u}\right)^{2},
$$

where $\delta>$ is some arbitrary small constant, and $c$ depends on $\delta$ and the maximum of $\alpha_{1}(u), \sigma_{0}(u)$ and $\sigma_{1}(u)$ for $t \leq u \leq T$, that is, the maximum of $h_{1}(u)$ and $h_{2}(u)$ for $t \leq u \leq T$.

Hence the first expectation on the right-hand side in (4.7) can be majorized by

$$
\begin{aligned}
\mathbb{E}^{t, s}[\exp & \left\{4(1+\epsilon) \gamma \int_{t}^{\tau}\left(\alpha_{1}(u)-\sigma_{0}(u) \sigma_{1}(u)\right) \ln S_{u}\right. \\
& \left.\left.+\left(\alpha_{2}(u)-\frac{1}{2} \sigma_{1}^{2}(u)\right)\left(\ln S_{u}\right)^{2} d u\right\}\right]^{1 / 2} \\
\leq c \mathbb{E}^{t, s} & {\left[\exp \left\{4(1+\epsilon) \gamma\left(\delta+\frac{\alpha^{2}}{\sigma^{2}(1-\gamma)}\right) \int_{t}^{T}\left(\ln S_{u}\right)^{2} d u\right\}\right]^{1 / 2} }
\end{aligned}
$$

where we have used that $\alpha_{1}(u)-0.5 \sigma_{1}^{2}(u) \leq \alpha^{2} / \sigma^{2}(1-\gamma)$.

Appealing to Doob's martingale inequality (see e.g. Protter [7, Thm. 20]) in the second expectation, we find,

$$
\begin{aligned}
& \mathbb{E}^{t, s}[\exp \left.\left\{4(1+\epsilon) \gamma \int_{t}^{\tau}\left(\sigma_{0}(u)+\sigma_{1}(u) \ln S_{u}\right) d B_{u}\right\}\right]^{1 / 2} \\
& \leq 2 \sup _{t \leq s \leq T} \mathbb{E}^{t, s}\left[\exp \left\{4(1+\epsilon) \gamma \int_{t}^{s}\left(\sigma_{0}(u)+\sigma_{1}(u) \ln S_{u}\right) d B_{u}\right\}\right]^{1 / 2}
\end{aligned}
$$

Subtracting and adding $16(1+\epsilon)^{2} \gamma^{2} \int_{t}^{s}\left(\sigma_{0}(u)+\sigma_{1}(u) \ln S_{u}\right)^{2} d u$ in the exponential, and then invoking the Cauchy-Schwartz inequality gives us from the martingale 
property that

$$
\begin{aligned}
\mathbb{E}^{t, s} & {\left[\exp \left\{4(1+\epsilon) \gamma \int_{t}^{\tau}\left(\sigma_{0}(u)+\sigma_{1}(u) \ln S_{u}\right) d B_{u}\right\}\right]^{1 / 2} } \\
& \leq 2 \mathbb{E}^{t, s}\left[\exp \left\{32(1+\epsilon)^{2} \gamma^{2} \int_{t}^{T}\left(\sigma_{0}(u)+\sigma_{1}(u) \ln S_{u}\right)^{2} d s\right\}\right]^{1 / 4} \\
& \leq 2 c \mathbb{E}^{t, s}\left[\exp \left\{32(1+\epsilon)^{2} \gamma^{2} \int_{t}^{T}\left(\delta+\sigma_{1}^{2}(u)\right)\left(\ln S_{u}\right)^{2} d u\right\}\right]^{1 / 4}
\end{aligned}
$$

where $\delta>0$ is an arbitrary positive constant and $c$ is dependent on the maximum of $\sigma_{0}(u)$. Now, since $h_{2}(u)$ is nonincreasing, positive, and $h_{2}(T)=0$, we find that

$$
\sigma_{1}^{2}(u) \leq \frac{4 \sigma^{2}}{(1-\gamma)^{2}} h_{2}(t)+\frac{\alpha^{2}}{\sigma^{2}(1-\gamma)^{2}}
$$

Summing up, we need to show that

$$
\begin{aligned}
& \mathbb{E}^{t, w, s}\left[\left(W_{\tau}^{\star}\right)^{2(1+\epsilon) \gamma}\right] \\
& \leq c \mathbb{E}^{t, s}\left[\exp \left\{4(1+\epsilon) \gamma\left(\delta+\frac{\alpha^{2}}{\sigma^{2}(1-\gamma)}\right) \int_{t}^{T}\left(\ln S_{u}\right)^{2} d u\right\}\right]^{1 / 2} \\
& \quad \times \mathbb{E}^{t, s}\left[\exp \left\{32(1+\epsilon)^{2} \gamma^{2}\left(\delta+\frac{\alpha^{2}}{\sigma^{2}(1-\gamma)^{2}}+\frac{4 \sigma^{2}}{(1-\gamma)^{2}} h_{2}(t)\right) \int_{t}^{T}\left(\ln S_{u}\right)^{2} d u\right\}\right]^{1 / 4}
\end{aligned}
$$

is finite. This is true by Lemma 4.3 whenever

$$
32(1+\epsilon)^{2} \gamma^{2}\left(\delta+\frac{\alpha^{2}}{\sigma^{2}(1-\gamma)^{2}}+\frac{4 \sigma^{2}}{(1-\gamma)^{2}} h_{2}(t)\right)<\frac{\alpha}{2 \sigma^{2}(T-t)},
$$

and

$$
4(1+\epsilon) \gamma\left(\delta+\frac{\alpha^{2}}{\sigma^{2}(1-\gamma)}\right)<\frac{\alpha}{2 \sigma^{2}(T-t)}
$$

Conditions (4.3)-(4.4) ensure this.

We conclude that $v\left(\tau, W_{\tau}^{\star}, S_{\tau}\right)$ is in $L^{1+\epsilon}(P)$ uniformly with respect to all stopping times $t \leq \tau \leq T$, and thus the family $\left\{v\left(\tau, W_{\tau}^{\star}, S_{\tau}\right)\right\}_{\tau}$ is uniformly integrable. Now the theorem follows immediately from Propositions 4.1 and 4.2 .

Note that the investment strategy is dependent on time and the current price level of the risky asset, but not dependent on the level of wealth. We also notice that the optimal control can be rewritten as the following expression

$$
\pi^{\star}(t, s)=\frac{\alpha(\mu-\ln s)-r}{\sigma^{2}(1-\gamma)}+f_{1}(t)+2 f_{2}(t) \ln s,
$$

where $f_{1}$ and $f_{2}$ are given in (3.4) and (3.5), respectively. The first term in $\pi^{\star}$ is analogous to the classical Merton investment strategy [3], which says that it is optimal to invest a fraction in the risky asset proportional to the risk premium per unit risk, where the risk is measured in the square of the volatility. The proportionality factor is one over the risk aversion. The second term adjusts this investment behaviour according to time and spot level. Notice that the position in the risky asset varies from being long to short (or opposite) depending on $\ln s$. 
The conditions (4.2)-(4.4) on the parameters of the control problem ensuring that the value function can be identified (and that it indeed is finite) involves $\alpha$, $\sigma$ and $\gamma$, together with the investment horizon $[t, T]$. However, the mean level $\mu$ is not part of the condition. We consider an example which shows that the conditions in fact are satisfied for a natural choice of parameters. Benth and Saltyte-Benth [1] estimated $\alpha$ and $\sigma$ for a time series of daily spot prices of Brent oil to be 0.004 and 0.021 respectively. Hence, consider the control problem with $\alpha=0.004$ and $\sigma=0.021$. Assume that the investor has risk preferences given by $\gamma=1 / 2$, and that the investment period start at $t=0$. If we furthermore choose $\epsilon$ and $\delta$ to be small, of the order $10^{-8}$, we find that the first condition (4.2) is satisfied for $T \leq 195.1$, while the second restriction (4.3) holds for all $T$ such that $T \leq 31.2$. Finally, condition (4.4) holds for $T \leq 3.8$. Since the measurement scale for Brent oil data are daily, the admissible investment horizon is very short for the third condition, being only 3.8 days. This motivates a more detailed estimation procedure in the proof of Theorem 4.4 to sort out better conditions which allow for longer investment horizons. If we instead fix the investment horizon to be $T=250$ days, and ask which risk preferences $\gamma$ are allowed, we find (using the same choice of $\alpha$ and $\sigma$ ) that condition (4.2) holds for $\gamma \leq 0.31$, while conditions (4.3)-(4.4) are valid when $\gamma \leq 0.11$. We see that the conditions for uniform integrability put restrictions on either the investment period or the allowed risk preferences for which we can uniquely identify the value function and the allocation strategy for the portfolio problem.

Finally, in view of Proposition 4.2, we notice that conditions (4.2)-(4.4) are needed to uniquely identify our candidate solution (3.6) as the value function (2.4) on an investment horizon $[t, T]$. The number $T-t$, i.e., the size of the time interval $[t, T]$ on which the HJB equation (2.6) has a unique solution, depends on the given parameters of the control problem (see the example above). We stress that the underlying mechanism that restricts the size of $T-t$ is completely analogous to the well known blow-up/uniqueness condition for the initial value problem for the heat equation. For this problem, a unique solution exists up to a certain time for initial conditions having growth given by $\exp \left(a x^{2}\right)$. This time $T$ will depend on $a$, and the solution can be represented as a convolution product with the Green's function (which corresponds to an expectation of the initial condition evaluated at the state of a Brownian motion). After time $T$, this solution will explode, and there are infinitely many solutions that solves the heat equation.

\section{REFERENCES}

[1] F. E. Benth and J. Saltyte-Benth. The normal inverse gaussian distribution and spot price modeling in energy markets. Int. J. Theor. Appl. Finance, 7(2):177-192, 2004.

[2] L. Clewlow and C. Strickland. Energy Derivatives. Pricing and Risk Management. Lacima Publications, London, 2000.

[3] M. Merton. Lifetime portfolio selection under uncertainty: the continuous case. Rev. Econom. Studies, 51:247-257, 1969.

[4] M. Musiela and T. Zariphopoulou. An example of indifference prices under exponential preferences. Finance Stoch., 8(2):229-239, 2004.

[5] H. Pham. Smooth solutions to optimal investment models with stochastic volatilities and portfolio constraints. Appl. Math. Optim., 46(1):55-78, 2002.

[6] D. Pilipović. Energy Risk. Valuing and Managing Energy Derivatives. McGraw-Hill, New York, 1998 
[7] P. Protter. Stochastic integration and differential equations, volume 21 of Applications of Mathematics (New York). Springer-Verlag, Berlin, 1990. A new approach.

[8] E. S. Schwartz. The stochastic behaviour of commodity prices: Implications for valuation and hedging. J. Finance, LII(3):923-973, 1997.

[9] T. Zariphopoulou. Optimal investment and consumption models with non-linear stock dynamics. Math. Methods Oper. Res., 50(2):271-296, 1999. Financial optimization.

(Fred Espen Benth)

Centre of Mathematics for Applications (CMA)

Department of Mathematics

UNIVERSITY OF OSLO

P.O. BOX 1053, BLINDERN

N-0316 OsLo, Norway

AND

Agder University College

Department of Economics and Business Administration

SERVICEBOKS 422

N-4604 KRIstiansand, NoRWaY

E-mail address: fredb@math.uio.no

URL: http:// Hнr.math.uio.no/ fredb/

(Kenneth Hvistendahl Karlsen)

Centre of Mathematics for Applications (CMA)

Department of Mathematics

UNIVERSITY OF OSLO

P.O. BOX 1053, BLINDERN

N-0316 OsLo, NoRWay

E-mail address: kennethk@math.uio.no

URL: http://ннн.mi.uib.no/“kennethk/ 